\title{
Optimisation of energy harvesting for stiffened composite shells with application to the aircraft wing at structural flight frequency
}

\author{
${ }^{1}$ Ali H., Daraji \\ School of Mechanical, Aerospace and Automotive Engineering, Coventry University, Coventry, UK \\ 2Jack M., Hale \\ School of Engineering, Newcastle University, Newcastle upon Tyne, UK \\ ${ }^{3}$ Jianqiao Ye \\ Engineering Department, Lancaster University, Lancaster, UK \\ Email: ${ }^{1}$ ac7202@coventry.ac.uk, ${ }^{2}$ Jack.hale@newcastle.ac.uk, 3.j.ye2@lancaster.ac.uk
}

\begin{abstract}
The conversion of structural dynamic strain into electric power using piezoelectric transducers to power microelectronic devices and wireless sensor nodes for structure health monitoring has been receiving growing attention from academic researchers and industry. Harvesting electric energy from vibration and storing it in an external infinite life-span capacitor is a proposed technique to eliminate the drawbacks of using conventional finite life-span batteries. Optimisation of the harvested power is an important research topic to ensure an endless power source with sufficient flow of electricity.

This paper concerns optimisation of energy harvesting for composite shells stiffened by beams, with discrete flexible composite piezoelectric sensors bonded to the surface and located optimally. A homogenous composite shell stiffened by beams with a bonded piezoelectric transducer connected to an external resistive load is modelled using three-dimensional solid finite elements. An efficient and effective placement methodology is proposed to find the optimal locations of piezoelectric sensors based on the maximisation of average percentage sensor effectiveness as an objective function. This study is firstly verified against published work for a cantilever flat plate and beam, and then implemented to optimise the energy harvesting for a composite aircraft wing at structural frequencies during flight. The results show a high reduction in computational effort and improved effectiveness of the methodology to optimise energy harvesting for complex and large-scale structures compared with alternative methods. Furthermore, the harvesting power obtained from optimal sensor distribution shows promise to be sufficient to activate wireless sensor nodes for health monitoring.
\end{abstract}

Key words: Aircraft wing, thin-walled composite shell, flexible piezoelectric, energy harvesting, vibration, optimisation. 


\section{Introduction}

Over the past decades, the manufacturing of wireless sensor nodes and microelectronic devices has been progressively developed to minimise size, power consumption and cost. This development has motivated academic researchers and companies to find and develop alternative power sources to eliminate the drawbacks of conventional batteries. These drawbacks are related to the size, weight, material cost, replacement cost, lifespan and suitability for deployment in toxic chemical and freezing environments. A focus has been placed on using piezoelectric energy harvesting systems as an alternative power source, stored in an external capacitor. A great many articles have been published on the development of energy harvesting systems in a wide range of engineering applications. One of the earliest studies was done by Umeda et al to investigate the feasibility of converting a mechanical impact into electric power using a piezoelectric transducer [1]. Song et al investigated small three-layer cantilever beams with tip masses and surfaces fully covered by macro fibre composite (MFC) patches vibrate at the same frequency to increase the energy harvesting [2]. Patel et al investigated the effects of a geometrical parameter for different configurations of coverage of piezoelectric on a cantilever beam energy harvester. The authors reported a significant increase in energy harvesting when reducing the piezoelectric layer length compared to the conventional full coverage [3]. The effects of nonlinearity of the piezoelectric response in electrostatic energy harvesting at resonant frequency were investigated for a cantilever beam entirely covered with piezoelectric pairs [4]. A cantilever beam with tip mass and bonded MFCs was investigated by Abdelkfi et al to optimise the energy harvesting based on the optimal external resistive load. Based on the analytical, finite element and experimental results, the authors reported that the beam model partially covered with piezoelectric on the basis of mode shapes gave better results than the beam fully covered by piezoelectric [5]. Amini et al used a Bernoulli beam model entirely covered by functionally graded piezoelectric harvesters to investigate the effects of unimorph, bimorph, series and parallel connection configurations [6]. Experimental analysis of energy harvesting of a cantilever composite beam with a single MFC bonded to its surface was investigated by Zakaria et al to find the effect of angle of attack, wind speed and load resistance on the level of the harvested power [7].

Yang et al studied the energy harvester for a cantilever beam with single, double and triple MFC segments at the root of the beam with the segments electrically connected in series and parallel, simulated using finite element analysis and tested experimentally. The authors noted that the parallel connection generated larger charging currents than series [8]. Finite element modelling presented by Vieira et al. for a thin plate bonded with piezoelectric based on classical plate theory and the double lattice method. The modelling was applied for a small flat plate-like wing bonded with two separated piezoelectric segments at the root of each plate sides were considered to prevent power cancellation at torsional modes and tested in a wind tunnel [9]. De Marqui et al investigated a small flat plate-like wing partially bonded with piezoelectric in two configurations as continuous and discrete segments under airflow excitation. Using time-domain analysis, the authors showed that the peak power generated by the segmented piezoelectric sensors was larger than the continuous and prevented power cancellation [10] and frequency domain analysis led to a reduction in the computational effort [11]. 
The optimisation of energy harvesting is a crucial research topic to ensure a constant supply of electricity. It is mainly based on the dimensions of the piezoelectric sensor, position on the structure and the resistive load of microelectronic devices connected between the sensor's electrodes. Sensor area and location have a significant effect on the amount of power harvested, but the large surface area does not guarantee high voltage and power generation since there is a cancelling effect when a sensor covers regions of tensile and compressive strains. Similarly, discrete piezoelectric sensors misplaced in regions of low strain or located randomly are likely to generate lower voltage [12].

Energy harvesting has been investigated for a cantilever plate-like wing with a single piezoelectric sensor bonded to it at the root [9-11]. These studies showed a cancellation of the harvested power at the torsional modes [11], but the cancellation phenomenon was eliminated by splitting the piezoelectric sensor into two parts $[9,10]$. The voltage and power are reduced and cancelled out for structures with a bonded piezoelectric sensor covering regions of negative and positive strain at the same time [13-15]. Similarly, the optimisation of sensing and actuating effects have been investigated thoroughly for active vibration control of simple and complex structures by the effective location of discrete piezoelectric sensors and actuators [12].

Most published studies investigating energy harvesting for aircraft wings employ small flat beam or plate structures with piezoelectric sensors bonded to the surface with entire or partial coverage [9-11, 16-18]. Based on the authors' best knowledge, there is a lack of investigation in the optimal distribution of discrete piezoelectric sensors to harvest energy optimally from a more realistic aircraft wing model and flexible structures. This was partly due to the complexity of the models and the high computation cost for identifying the optimal sensor locations. In this study, an effective and efficient piezoelectric sensor placement methodology is proposed for maximisation of the sensor voltage and power. A composite shell stiffened by beams with bonded piezoelectric sensors is modelled using three-dimensional solid finite elements. This methodology is validated by optimisation of six piezoelectric sensors for a cantilever plate and then the placement methodology was employed to maximise energy harvesting for a wing structure stiffened by spars and ribs.

\section{MODELLING}

\subsection{Finite element modelling}

Aircraft wings are mostly constructed as thin-walled shells stiffened by spars and ribs to optimise the specific strength, loading capacity and fuel consumption. These structures are associated with complex vibration modes, which can cause failure as a result of internal or external micro-cracking. Continuous observation and online monitoring, which are normally powered by batteries, for early detection of micro-cracks before failure is required to save cost, maintenance and lives. In this study, piezoelectric sensors are located optimally to harvest electric power from dynamic strain at structural flight frequencies, which may provide continuous energy supplies to power wireless sensor nodes for the monitoring and crack detection. Harvesting energy in aerospace structures is considered a perfect solution to eliminate the drawbacks of conventional batteries in powering wireless sensor nodes. 
The modelling considers a composite shell stiffened by beams with a perfectly bonded piezoelectric sensor, as shown in Figure 1. The laminated composite shell and the stiffeners are assumed to be anisotropically homogenous, and the structural mass, stiffness, damping and piezoelectric coefficients are supposed to be timeinvariant and linear elastic. Twenty node isoparametric three-dimensional solid elements are used in modelling to discretise the shell, stiffener and sensor. The displacements of the 3D solid element $u, v, w$ are related to the linear nodal displacements $u_{i}, v_{i}, w_{i}$ and shape function $N_{i}(s, r, t)$ according to eq (1) below, where the node number $i=1-20$. The shape function represents the element geometry and the natural coordinates $s, r$ and $t$, varying between -1 and 1 .

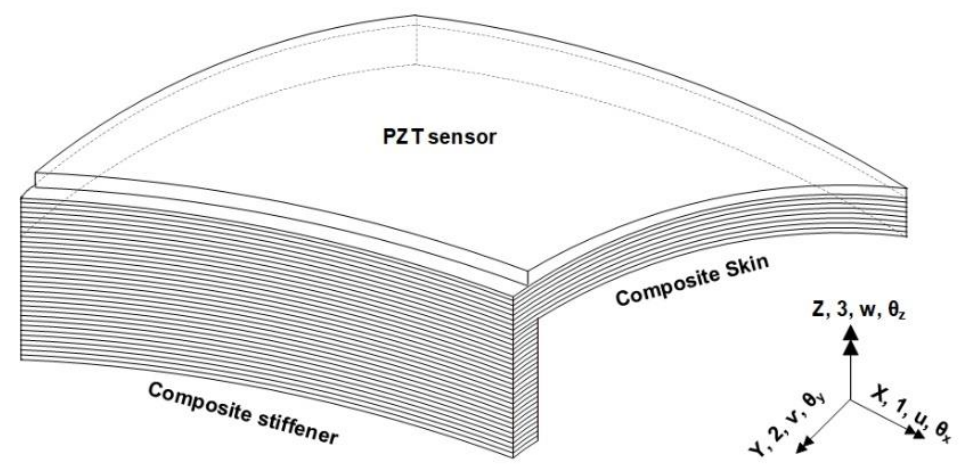

Figure 1. Wing skin element stiffened by beam bonded piezoelectric sensor

$$
\begin{aligned}
& \left\{\begin{array}{l}
u \\
v \\
w
\end{array}\right\}=\sum_{i=1}^{20} N_{i}(s, r, t)\left\{\begin{array}{l}
u_{i} \\
v_{i} \\
w_{i}
\end{array}\right\}=\sum_{i=1}^{20} N_{i} \boldsymbol{\delta}_{i} \\
& \boldsymbol{\varepsilon}=\left\{\begin{array}{l}
\varepsilon_{x} \\
\varepsilon_{y} \\
\varepsilon_{z}
\end{array}\right\}+\left\{\begin{array}{l}
\gamma_{x y} \\
\gamma_{y z} \\
\gamma_{z x}
\end{array}\right\}=\left\{\begin{array}{l}
u, x \\
v, y \\
w, z
\end{array}\right\}+\left\{\begin{array}{l}
u, y+v, x \\
v, z+w, y \\
w, x+u, z
\end{array}\right\}=\sum_{i=1}^{20} B_{i} \boldsymbol{\delta}_{i} \\
& \boldsymbol{B}_{i}=\left[\begin{array}{ccc}
N_{i}, x & 0 & 0 \\
0 & N_{i}, y & 0 \\
0 & 0 & N_{i}, z \\
N_{i}, y & N_{i}, x & 0 \\
0 & N_{i}, z & N_{i}, y \\
N_{i}, z & 0 & N_{i}, x
\end{array}\right]
\end{aligned}
$$

$\boldsymbol{\delta}=\left\{\begin{array}{llll}\boldsymbol{\delta}_{1} & \boldsymbol{\delta}_{2} & \ldots & \boldsymbol{\delta}_{20}\end{array}\right\}^{\mathrm{T}}, \boldsymbol{\delta}_{i}=\left\{\begin{array}{lll}u_{i} & v_{i} & w_{i}\end{array}\right\}^{\mathrm{T}}$

$\boldsymbol{\sigma}=\left\{\begin{array}{llllll}\sigma_{x x} & \sigma_{y y} & \sigma_{z z} & \tau_{x y} & \tau_{y z} & \left.\tau_{z x}\right\}^{T}=\boldsymbol{D} \boldsymbol{\varepsilon}\end{array}\right.$ 
Where $\boldsymbol{B}$ is a $6 \times 60$ differential matrix relating the element strains $\boldsymbol{\varepsilon}$ at coordinate $(x, y, z, t)$ to the element nodal displacements $\boldsymbol{\delta} ; \mathbf{N}$ is $6 \times 60$ shape function matrix represents the element geometry and relates the element displacement $\{u v w\}^{T}$ at coordinate $(x, y, z, t)$ to the element nodal displacement $\boldsymbol{\delta} . \boldsymbol{\sigma}$ and $\boldsymbol{D}$ denote the element stresses and the elasticity matrix, respectively.

\subsection{Piezoelectric constitutive equation}

Piezoelectric material is an essential element in a smart structure to detect and actuate vibration. It generates a voltage when subjected to dynamic strain and vice versa. The piezoelectric ceramic material leadzirconate-titanate (PZT) has received much attention in sensing and actuating applications because it has mechanical simplicity, small volume, lightweight, large useful bandwidth, efficient conversion between mechanical and electrical energy and easy integration with various metallic and composite structures [19]. Solid ceramic PZT is a very effective piezoelectric material, but is brittle and requires extra attention during the handling and bonding procedures. Furthermore, the adaptability to a curved surface is extremely poor, requiring further treatment of the surfaces and additional manufacturing capabilities. These restrictions are eliminated by the development of a composite piezoelectric transducer called macro fibre composite piezoelectric sensor, such as the MFCs and the DuraAct piezoelectric transducer. It is flexible and adaptable to the curved surface and so is suitable for bonding on aircraft structures. The linear constitutive equations of a piezoelectric material relating stresses, $\boldsymbol{\sigma}$, and electric displacement, $\boldsymbol{D}_{e}$ to the strains, $\boldsymbol{\varepsilon}$, and the electric field, $\boldsymbol{E}_{f}$, are presented in eq. (6).

$$
\left\{\begin{array}{c}
\boldsymbol{\sigma} \\
\boldsymbol{D}_{\boldsymbol{e}}
\end{array}\right\}=\left[\begin{array}{cc}
\boldsymbol{C}^{\mathrm{E}} & -\boldsymbol{e}^{\mathrm{T}} \\
\boldsymbol{e} & \boldsymbol{\mu}^{\sigma}
\end{array}\right]\left\{\begin{array}{c}
\boldsymbol{\varepsilon} \\
\boldsymbol{E}_{f}
\end{array}\right\}
$$

where $\boldsymbol{C}, \boldsymbol{e}$, and $\boldsymbol{\mu}$ are elasticity, piezoelectric and permittivity matrices. The superscripts $E$ and $\sigma$ denote that the measurements are taken under constant electrical displacement and stress, respectively.

\subsection{Hamilton's principle}

Hamilton's principle is a variational principle based on the variation of energy scalar quantities. It is a simple and powerful tool used to derive the dynamic equilibrium equations used in this study. It was used by Tzou and Tseng for active vibration control of a smart plate [20] and is used here to model the stiffened shell element bonded with a piezoelectric sensor. Hamilton's principle states that the variational integral must equal to zero at all times, as shown in eq. (7).

$$
V I=\int_{\mathrm{t} 1}^{\mathrm{t} 2}(\Delta \mathrm{T}-\Delta \mathrm{U}+\Delta \mathrm{W}) \mathrm{dt}=0
$$

where $\mathrm{T}, \mathrm{U}$ and $\mathrm{W}$ are the time-dependent kinetic energy, strain energy including piezoelectric energy and external applied work. The respective kinetic energy induced in the shell, the sensor and the stiffener are:

$$
T=0.5 \rho \int\left(\dot{u}^{2}+\dot{v}^{2}+\dot{w}^{2}\right) d v=0.5 \dot{\boldsymbol{\delta}}^{\mathrm{T}} \boldsymbol{m} \dot{\boldsymbol{\delta}}
$$




$$
\begin{aligned}
& T=0.5 \dot{\boldsymbol{\delta}}^{\mathrm{T}}\left[\boldsymbol{m}_{s l}+\boldsymbol{m}_{s t}+\boldsymbol{m}_{s}\right] \dot{\boldsymbol{\delta}}=0.5 \dot{\boldsymbol{\delta}}^{\mathrm{T}} \boldsymbol{M} \dot{\boldsymbol{\delta}} \\
& \Delta T=\dot{\boldsymbol{\delta}}^{\mathrm{T}} \boldsymbol{M} \Delta \dot{\boldsymbol{\delta}}
\end{aligned}
$$

Where, Superscripts $s l$, st and $s$ refer to shell, stiffener and sensor, respectively. The total strain energy $U$ induced in a shell with stiffener and sensor, including the electrical energy, can be described by the following equation:

$$
U=\frac{1}{2} \int_{v} \boldsymbol{\varepsilon}^{\mathrm{T}} \boldsymbol{\sigma} d v-\frac{1}{2} \int_{v} \boldsymbol{E}_{f}^{\mathrm{T}} \boldsymbol{D}_{e} d v_{s}
$$

The distribution of the electrical field, $\boldsymbol{E}_{f}$ varies linearly across the thickness of a piezoelectric element $h_{s}$, and the voltage difference across its thickness is constant over the entire element. Hence,

$$
\begin{aligned}
\boldsymbol{E}_{f} & =\left\{\begin{array}{lll}
E_{1} & E_{2} & E_{3}
\end{array}\right\}^{T} \\
\boldsymbol{E}_{f} & =-\frac{\phi}{h_{s}}\left\{\begin{array}{lll}
1 & 1 & 1
\end{array}\right\}^{T}=-\boldsymbol{B}_{\varnothing} \phi
\end{aligned}
$$

where $\phi$ is the voltage induced over the piezoelectric electrodes. By substituting eqs (5), (6) and (13) into eq. (11), the following equations are obtained:

$$
\begin{aligned}
& U=0.5 \int_{v} \boldsymbol{\delta}^{T}\left(\boldsymbol{B}^{T} \boldsymbol{D} \boldsymbol{B} \boldsymbol{\delta} d v_{s l}+\boldsymbol{B}^{T} \boldsymbol{D} \boldsymbol{B} \boldsymbol{\delta} d v_{s t}+\boldsymbol{B}^{T} \boldsymbol{C}^{E} \boldsymbol{B} \boldsymbol{\delta} d v_{s}+\boldsymbol{B}^{T} \boldsymbol{e}^{T} \boldsymbol{B}_{\emptyset} \boldsymbol{\phi} d v_{s}+\boldsymbol{B}_{\varnothing}^{\mathrm{T}} \boldsymbol{e} \boldsymbol{B} \boldsymbol{\phi} d v_{s}\right) \\
& -\boldsymbol{\phi}^{T} \boldsymbol{B}_{\varnothing}^{\mathrm{T}} \boldsymbol{\mu}^{\sigma} \boldsymbol{B}_{\varnothing} \boldsymbol{\phi} d v_{s} \\
& U=0.5\left(\boldsymbol{\delta}^{\mathrm{T}} \boldsymbol{K}_{u \boldsymbol{u}}^{\boldsymbol{s l}} \boldsymbol{\delta}+\boldsymbol{\delta}^{\mathrm{T}} \boldsymbol{K}_{u \boldsymbol{u}}^{s t} \boldsymbol{\delta}+\boldsymbol{\delta}^{\mathrm{T}} \boldsymbol{K}_{u \boldsymbol{u}}^{\boldsymbol{s}} \boldsymbol{\delta}+\boldsymbol{\delta}^{\mathrm{T}} \boldsymbol{K}_{u \emptyset}^{s} \boldsymbol{\phi}+\boldsymbol{\phi}^{\mathrm{T}} \boldsymbol{K}_{\emptyset u}^{s} \boldsymbol{\delta}+\boldsymbol{\phi}^{\mathrm{T}} \boldsymbol{K}_{\emptyset \emptyset}^{s} \boldsymbol{\phi}\right) \\
& U=0.5\left(\boldsymbol{\delta}^{\mathrm{T}} \boldsymbol{K} \boldsymbol{\delta}+\boldsymbol{\delta}^{\mathrm{T}} \boldsymbol{K}_{u \emptyset}^{s} \boldsymbol{\phi}+\boldsymbol{\phi}^{\mathrm{T}} \boldsymbol{K}_{\emptyset u}^{s} \boldsymbol{\delta}+\boldsymbol{\phi}^{\mathrm{T}} \boldsymbol{K}_{\emptyset \emptyset}^{s} \boldsymbol{\phi}\right) \\
& \boldsymbol{K}=\boldsymbol{\delta}^{\mathrm{T}}\left(\boldsymbol{K}_{u u}^{s l}+\boldsymbol{K}_{u u}^{s t}+\boldsymbol{K}_{u u}^{s}\right) \boldsymbol{\delta} \\
& \Delta U=\Delta \boldsymbol{\delta}^{\mathrm{T}} \boldsymbol{K} \boldsymbol{\delta}+\Delta \boldsymbol{\delta}^{\mathrm{T}} \boldsymbol{K}_{u \varnothing}^{s} \boldsymbol{\phi}+\Delta \boldsymbol{\phi}^{\mathrm{T}} \boldsymbol{K}_{\emptyset u}^{s} \boldsymbol{\delta}+\Delta \boldsymbol{\phi}^{\mathrm{T}} \boldsymbol{K}_{\varnothing \varnothing}^{s} \boldsymbol{\phi}
\end{aligned}
$$

where $\boldsymbol{K}$ denotes the structural stiffness matrices for shell, stiffeners and sensors. The terms $\boldsymbol{K}_{\emptyset u}^{s}, \boldsymbol{K}_{\varnothing \emptyset}^{s}$ refer to the electromechanical coupling and the capacitance of the piezoelectric sensor. The work done by the external excitation force is given by:

$$
\Delta W=\Delta \boldsymbol{\delta}^{\mathrm{T}} \boldsymbol{F}_{\boldsymbol{u}}-\Delta \boldsymbol{\phi}^{\mathrm{T}} \boldsymbol{F}_{\phi}
$$

where $\boldsymbol{F}_{u}$ and $\boldsymbol{F}_{\phi}$ refer to the external excitation force applied to the structure and the piezoelectric sensor charge, respectively. By substituting eqs. (10), (18) and (19) into eq. (7) the following equation is obtained:

$$
\begin{gathered}
V I=\int_{\mathrm{t} 1}^{\mathrm{t} 2}\left(-\Delta \boldsymbol{\delta}^{\mathrm{T}} \boldsymbol{M} \ddot{\boldsymbol{\delta}}-\Delta \boldsymbol{\delta}^{\mathrm{T}} \boldsymbol{K} \boldsymbol{\delta}-\Delta \boldsymbol{\delta}^{\mathrm{T}} \boldsymbol{K}_{u \varnothing} \boldsymbol{\phi}-\Delta \boldsymbol{\phi}^{\mathrm{T}} \boldsymbol{K}_{\emptyset u} \boldsymbol{\delta}-\Delta \boldsymbol{\phi}^{\mathrm{T}} \boldsymbol{K}_{\varnothing \varnothing} \boldsymbol{\phi}+\Delta \boldsymbol{\delta}^{\mathrm{T}} \boldsymbol{F}_{\boldsymbol{u}}-\Delta \boldsymbol{\phi}^{\mathrm{T}} \boldsymbol{F}_{\phi}\right) \mathrm{dt} \\
=0
\end{gathered}
$$

The integration of the vibrational integral gives the following two equations:

$$
M \ddot{\delta}+K \delta+K_{u \emptyset}^{s} \phi=F_{u}
$$




$$
\boldsymbol{K}_{\emptyset \emptyset}^{s} \boldsymbol{\phi}+\boldsymbol{K}_{\emptyset u}^{s} \boldsymbol{\delta}=\boldsymbol{F}_{\phi}
$$

The Rayleigh structural damping force $\boldsymbol{C} \dot{\boldsymbol{\delta}}$ as a result of mass and stiffness damping effects can be added to eq. (21), as follows:

$$
\boldsymbol{M} \ddot{\boldsymbol{\delta}}+\boldsymbol{C} \dot{\boldsymbol{\delta}}+\boldsymbol{K} \boldsymbol{\delta}+\boldsymbol{K}_{u \emptyset}^{s} \boldsymbol{\phi}=\boldsymbol{F}_{\boldsymbol{u}}
$$

The solutions of the two coupled eqs. (22) and (23) give the structural motion and the piezoelectric voltage time responses. These equations show that the mechanical energy induced by an external excitation force would be dissipated entirely through structural damping and dynamic movement of the piezoelectric sensor. Part of the mechanical energy could be converted to electric power by connecting an external resistive load $R$ between the piezoelectric sensor electrodes. According to Ohm's law, the voltage drop across a connected external resistive is equal to the current flow times the resistance $R$ as given by the following formula:

$$
\phi=-R \dot{F}_{\phi}=-R i, \quad \dot{F}_{\phi}=i
$$

where $i$ is the current flow through the external resistive load. Differentiating eq.(22), then substituting eq. (24) into eqs (22) and (23) yields the following dynamic equilibrium equations for the energy harvesting system:

$$
\begin{aligned}
& \boldsymbol{M} \ddot{\boldsymbol{\delta}}+\boldsymbol{C} \dot{\boldsymbol{\delta}}+\boldsymbol{K} \boldsymbol{\delta}-\boldsymbol{K}_{u \emptyset}^{s} R i=\boldsymbol{F}_{\boldsymbol{u}} \\
& \frac{\phi}{R}+C_{s} \dot{\phi}+\boldsymbol{K}_{\emptyset u}^{s} \dot{\boldsymbol{\delta}}=0
\end{aligned}
$$

The generated average harvesting power by a piezoelectric sensor is equal to the product of the root mean square ( $\mathrm{rms}$ ) value of the current flow $i$ through the connected external load resistance $R$ between the piezoelectric sensor electrodes by the rms value of the voltage $\phi$ drop across the resistance. The generated average harvesting power $P_{a v}$ is represented by eq. 29 [21-23], as follows:

$$
\begin{aligned}
& \phi=\phi_{o} \sin (\omega t) \quad, \quad i=\frac{\phi_{o} \sin (\omega t)}{R}=i_{0} \sin (\omega t) \\
& \phi_{r m s}=\frac{\phi_{o}}{\sqrt{2}}, \quad i_{r m s}=\frac{i_{0}}{\sqrt{2}}=\frac{1}{\sqrt{2}} \frac{\phi_{o}}{R} \\
& P_{a v}=i_{r m s} \phi_{r m s}=0.5 \phi_{o}^{2} / R
\end{aligned}
$$

In this study, the piezoelectric harvesting power is determined based on the optimum average harvesting power, according to eq. 32. The harvesting power reaches the optimum value when the connected external load resistance is equal to the piezoelectric equivalent internal impedance [21,23]; in this case, the resistive load is called optimal resistance. The piezoelectric capacitance $C_{s}$, optimal resistive load $R_{\text {opt }}$, and the optimum average harvesting power are calculated as follows:

$$
C_{s}=\mu_{33}^{\sigma} \times \mu_{0} \times A / t
$$




$$
\begin{aligned}
& R_{o p t}=1 / 2 \pi f C_{s} \\
& P=0.5 \phi_{o}^{2} / R_{o p t}
\end{aligned}
$$

where $\phi_{o}$ and $i_{0}$ are the amplitude of the sinusoidal voltage $\phi$ dropped across the resistance and current flow $i$ flow through the resistance load $R$, and $\mu_{33}^{\sigma}, \mu_{0}, A$ and $t$ denote to piezoelectric relative permittivity, vacuum permittivity, surface area and thickness of the piezoelectric sensor.

\section{SENSOR PLACEMENT METHODOLOGY}

In this study, a generalised piezoelectric sensor placement methodology to optimise the energy harvesting of a flexible structure is proposed, based on the maximisation of the average percentage sensors effectiveness as an objective function. The percentage sensors effectiveness is defined as the percentage of the ratio of the voltage amplitude generated by a piezoelectric sensor located at a position on the structure surface to the maximum generated voltage amplitude by a specific sensor located on the structure at the same mode of vibration. The average percentage effectiveness of the sensor is considered as a fitness function to measure the goodness of the sensor position. The piezoelectric energy harvesting is optimised based on determination of the optimum position of the sensors on the surface of a structure and external resistance load connected between sensor electrodes. However, the number and the dimensions of the piezoelectric segments required to be optimised are preselected by the designer.

The distribution of sensors percentage effectiveness on a structure surface is constant for a given structural and piezoelectric geometry, dimensions, materials properties, boundary conditions and excitation frequency. These factors affect the sensor voltage and percentage effectiveness, and are predefined before they are determined. The unequal dynamic energy exciting a structure has an effect on the generation of the sensor voltage amplitudes, but it does not affect the distribution of percentage sensor effectiveness since this is based on the ratio of sensor amplitude voltages and normalised to $100 \%$. The application of the generalised methodology has the following characteristics:

1. The placement methodology is realised by covering the whole structure surface with perfectly bonded discrete piezoelectric sensors $n_{s}$, as shown in Figure 2. The ANSYS Parametric Design Language (APDL) program has been developed to determine the generated voltage amplitude of the sensors and their percentage effectiveness. The APDL program starts to enter the model creation processor (prep7) to define the element types, materials, structure and piezoelectric sensors dimensions, element size, meshing, piezoelectric positive and negative electrodes and boundary conditions. The structure and piezoelectric sensors are modelled using three-dimensional solid95 elements for the substrate of the passive structure and solid226 elements for the piezoelectric sensors, respectively.

2. Enter the solution processor using (/Solu) command to apply harmonic analysis using $($ Antype,3) command, valid for structural, magnetic, and electrical degrees of freedom. In the harmonic analysis the structure is excited by either applied forces or base motion (oscillation of the mounting edges) at the $n_{m}$ vibration modes of interest. 
3. Enter the database results postprocessing using (/Post1) to get the peak amplitude voltage $\phi_{o}$ for all the sensors at each mode of vibration. Then, check all sensor voltage amplitudes to find the sensor position generating the highest voltage amplitude $\phi_{o m}$ at each vibration mode of interest.

4. The percentage effectiveness of sensors is determined at each mode of vibration by dividing the voltage amplitude of all sensors by the maximum amplitude voltage. The percentage sensor effectiveness is noralised to $100 \%$ and the expected results for each sensor are between zero and $100 \%$ at each mode of vibration, according to the following equation:

$$
S_{i, j}=\frac{\left|\phi_{o_{i, j}}\right|}{\left|\phi_{o_{j}}\right|} 100 \% \quad \text { where } i=1,2,3 \ldots n_{s}, j=1,2,3 \ldots n_{m}
$$

where $S$ : percentage sensor effectiveness

$\phi_{o}$ : sensor voltage amplitude for time-domain analysis or peak sensor voltage for frequencydomain analysis. The computational effort for frequency-domain is much lower than timedomain analysis.

$\phi_{\text {om }}$ : maximum value of sensor voltage amplitude at specific mode

$n_{s}$ : total number of sensors covering a structure.

$n_{m}$ : total number of modes to be investigated

$i$ : represent the sensor position in the $x$ and $y$ direction on the structure surface.

$j$ : represent the vibration mode number

5. The average sensor percentage effectiveness $A S$ is considered as a fitness function to measure the goodness of each sensor. The expected result of $A S$ for each sensor is also in the range zero to $100 \%$ for multiple modes of vibration, calculated according to the following equation.

$$
A S_{i}=\frac{1}{n_{m}} \sum_{j=1}^{n_{m}} S_{i, j}
$$

6. Two numbers mark each sensor, the first defining the sensor position in $x$ and $y$ directions on the structure surface, and the second the average percentage effectiveness. The objective function is based on the maximisation of the average percentage effectiveness, with the high average percentage effectiveness sensors being selected as the optimum sensors as follows.

$$
J_{\text {opt }}(x, y)=\max \left(\sum_{i=1}^{n_{o}} A S_{i}\right) \quad x, y \in \text { structure dimesions }
$$

7. The number of active sensors $\gamma_{j}$ at each mode of vibration is determined from equation (36). This number is less than the total number of piezoelectric sensors available to be optimised to harvest energy at the same mode.

$$
\gamma_{j}=0.01 \sum_{i=1}^{n_{o}} S_{i, j}
$$


The total active sensors to harvest power at all the required modes of vibration is higher than the number of sensors required to be optimised and can be determined according to the following equation:

$$
\gamma=\sum_{i=1}^{n_{m}} \gamma_{j}
$$

\section{RESEARCH PROBLEM}

The composite aircraft wing to be studied in this paper is constructed as a composite shell stiffened by four spars and ten ribs, as shown in Figure 2 (a). The wing structure represents an optimisation problem for a large scale structure with significant complexity in geometry and optimisation. The composite stiffened shell is made of laminated carbon fibre composites (T300/5208) with a section profile of [04/454/904] with $5 \mathrm{~mm}$ skin thickness and $10 \mathrm{~mm}$ stiffener depth. The skin surface of the aircraft wing was discretised into 660 sub-areas of $22 \times 30$, each of which was fully covered with bonded flexible DuraAct piezoelectric sensor segments of $90 \times 40 \times 0.18 \mathrm{~mm}$, as shown in Figure 2 (b).
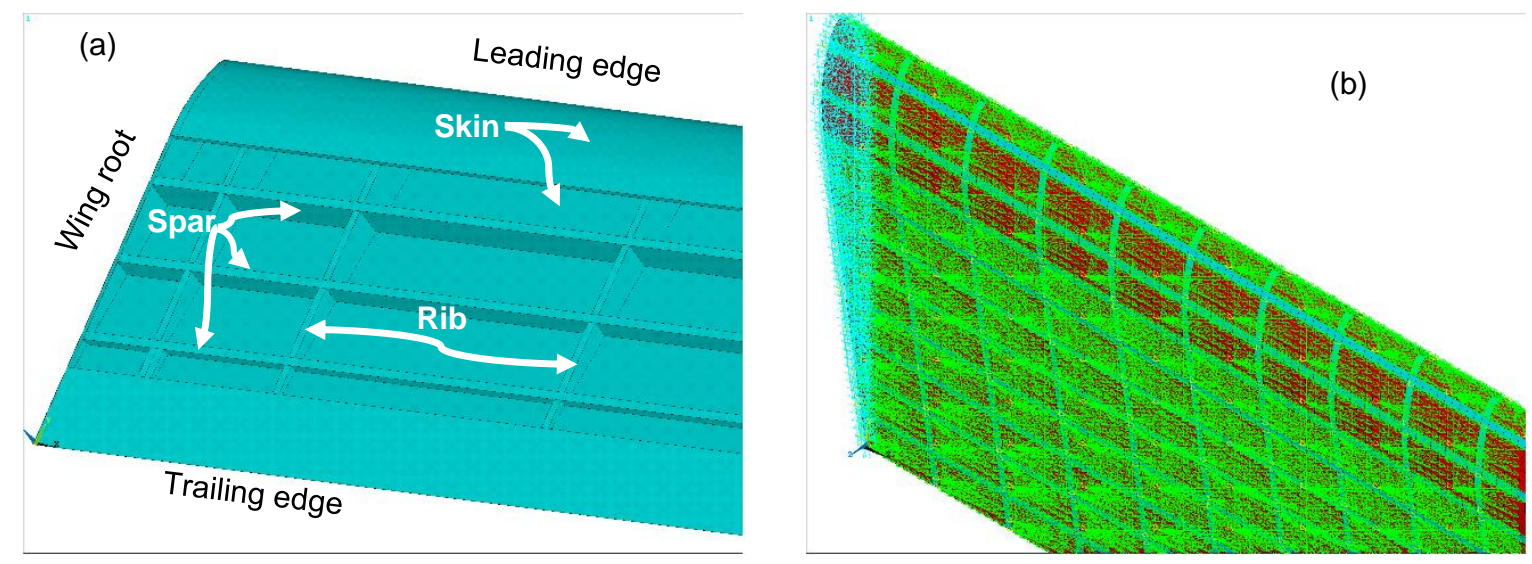

Figure 2. (a) Aircraft composite wing structure, (b) Aircraft composite wing structure bonded with full coverage of 660 discrete DuraAct piezoelectric sensors (red, green and blue colours denote to discrete piezoelectric sensors, points of voltage measure on sensor electrodes and wing structure respectively)

Table 1 shows the properties of the composite wing structure and the piezoelectric sensor. The aircraft wing was mounted rigidly along the entire root and subjected to external excitation at a structural flight frequency condition of $4-10 \mathrm{~Hz}$ generating maximum structural strain amplitude of $315 \mu$, which is well below the maximum permissible dynamic strain amplitude of $600 \mu$ of the piezoelectric sensor, and within the aircraft structure flight condition $[17,24]$. The proposed placement methodology explained in Section 3 is applied to obtain the optimal distribution based on the maximisation of sensor output voltages and their effectiveness. 
Table 1 Composite wing structure and DuraAct Piezoelectric sensor properties

\begin{tabular}{lll}
\hline Properties & Wing structure & DuraAct Piezo PIC255 \\
\hline$E_{x}, E_{y}, E_{z}$ (GPa) & $51.76,46.54,9.68$ & -------- \\
$G_{x y}, G_{y z}, G_{x z}$ (GPa) & $4.945,4.945,14.27$ & ------- \\
$\mu_{x y}, \mu_{y z}, \mu_{x z}$ & $0.475,0.155,0.153$ & ------- \\
Density $\left(\mathrm{Kg} / \mathrm{m}^{3}\right)$ & 1540 & 7800 \\
$e_{31}, e_{32}, e_{33}, e_{15}, \mathrm{C} / \mathrm{m}^{2}$ & -------- & $-7.15,-7.15,13.7,11.9$ \\
$C_{11}^{E}, C_{12}^{E}, C_{13}^{E}, \mathrm{GPa}$ & ------- & $123,76.7,70.25$ \\
$C_{22}^{E}, C_{23}^{E}, C_{33}^{E}$ & ------ & $123.11,70.2,97.11$ \\
$C_{44}^{E} C_{55}^{E}, C_{66}^{E}$ & ----- & $22.8,22.2,23.1$ \\
$\mu_{11}^{\sigma}, \mu_{22}^{\sigma}, \mu_{33}^{\sigma}$ & ------ & $930,930,870$ \\
\hline
\end{tabular}

\section{VALIDATION OF RESULTS.}

\section{1 optimal sensor placement}

To validate the proposed optimal piezoelectric sensor placement method, a composite cantilever plate was studied to select the six best sensor locations and the obtained results were compared with published results. The dimension of the composite plate is $180 \times 220 \times 0.82 \mathrm{~mm}$ discretised and bonded with ninety-nine piezoelectric sensors distributed to $9 \times 11$ locations and subjected to an external excitation force at the first five natural frequencies, as shown in Figure 3(a). An APDL program was designed based on the placement methodology explained in Section 3 to calculate the generated voltage and percentage effectiveness of the sensors. The results of the average percentage sensor effectiveness were mapped on the surface of the cantilever plate, as shown in Figure 3(a). The optimal placement of the six sensors on a cantilever plate was chosen on the basis of the ranking from Step 6 in Section 3, as indicated in red in Fig. 3(a). Figure 3 (b) shows the optimal placement of six sensors located by Han and Lee for the same cantilever plate using genetic algorithms based on gramian observability as an objective function to observe and suppress the first five modes of vibration [25]. The optimal locations of the six sensors from the present work agree with the published work shown in Figure $3(b)$ at four sensor locations and are different at two locations, but these two locations show a higher percentage of effectiveness for the current study.

Table 2 shows the results of analysis carried out for the optimal sensor configurations in Figure 3, comparing the current optimal sensor configuration with the configuration obtained in [25]. The table shows the contribution of the average percentage sensor effectiveness for each single-mode and all modes of vibration. Generally, the two sensor configurations achieved similar levels of sensor effectiveness in all modes, with similarly high values for all modes except the fifth. However, the configuration of the present method performed somewhat better in all modes. Table 2 shows the number of sensors and the total sensors that are actively involved, respectively, in each individual and all modes of vibration. 


\begin{tabular}{|l|l|l|l|l|l|l|l|l|l|l|}
\hline 73.4 & 38.7 & 19.9 & 24.4 & 27.9 & 28.5 & 26.3 & 21.2 & 13.8 & 7.4 & 5.2 \\
\hline 72.9 & 40.6 & 21.5 & 26.4 & 30.6 & 31.8 & 30.1 & 24.3 & 16.4 & 10.5 & 9.7 \\
\hline 64.5 & 36.4 & 21.5 & 26.4 & 31.4 & 33.4 & 32.5 & 28.1 & 21.7 & 16.7 & 16.4 \\
\hline 54.6 & 31.3 & 20.3 & 25.2 & 30.7 & 33.3 & 33.1 & 30.0 & 25.2 & 20.7 & 20.5 \\
\hline 54.2 & 25.4 & 18.1 & 22.5 & 27.9 & 30.6 & 30.6 & 28.5 & 25.2 & 21.8 & 21.4 \\
\hline 54.6 & 31.2 & 20.2 & 25.3 & 30.7 & 33.1 & 32.5 & 29.0 & 23.9 & 21.0 & 21.4 \\
\hline 72.2 & 36.0 & 21.4 & 26.6 & 31.4 & 33.1 & 31.8 & 26.9 & 20.0 & 16.2 & 16.7 \\
\hline 73.2 & 38.9 & 19.8 & 24.8 & 28.17 & 28.3 & 25.5 & 19.7 & 13.1 & 7.8 & 5.8 \\
\hline 14
\end{tabular}

(a), Present work

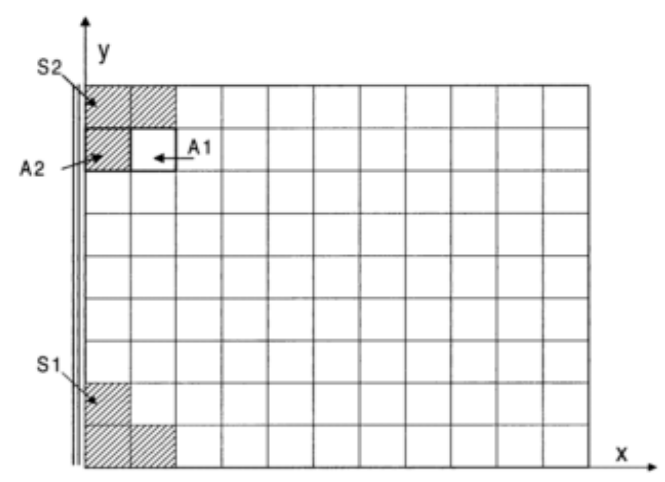

(b), [25 ]

Figure 3. Optimal distribution of six sensors on a composite cantilever plate

Table 2: Comparison of effectiveness of sensors on a cantilever plate

\begin{tabular}{|c|c|c|c|c|c|c|c|}
\hline \multirow{2}{*}{ Optimal sensor locations } & \multicolumn{6}{|c|}{ Average percentage effectiveness for all six sensors } & \multirow{2}{*}{$\begin{array}{l}\text { Total number of } \\
\text { active sensor for } \\
\text { each case study }\end{array}$} \\
\hline & $1^{\text {st }}$ & $2^{\text {nd }}$ & $3^{\text {rd }}$ & $4^{\text {th }}$ & $5^{\text {th }}$ & Average & \\
\hline Optimal /[25 ] & 68.9 & 81.4 & 71.5 & 72.9 & 13.0 & 61.5 & $0.61 \times 6 \times 5=18$ \\
\hline Optimal /Present study & 82.9 & 81.97 & 88.19 & 79.7 & 17.5 & 70.0 & $0.7 \times 6 \times 5=21$ \\
\hline $\begin{array}{l}\text { Number of active sensors } \\
\text { /present study }\end{array}$ & 4.97 & 4.91 & 5.29 & 4.78 & 1.05 & & \\
\hline
\end{tabular}

It can be observed that the optimal placement of the present method in this paper offers higher percentage effectiveness in all the five modes of vibration in comparison with the published optimal sensors configuration. A higher percentage sensor effectiveness indicates a higher voltage and power produced from a sensor at the first five modes of vibration. It is worth of mentioning that the degree of complexity of optimal placement using genetic algorithms is very high for such a simple structure. As reported by the Han and Lee [25] that the total number of candidate solutions for this cantilever plate were equal to all possible combinations of sensor locations, i.e., ${ }_{99} \mathrm{C}_{6}=11.2 \times 10^{9}$., which is too big for such a small plate. In contrast, the placement method proposed in this study is far more computational effective, which is particularly beneficial for larger-scale structures like aircraft fuselage or wings.

\subsection{Energy harvesting}

In the second validation of the proposed approach in this paper, energy harvesting from an aluminium cantilever beam with tip mass is investigated and compared with available simulation and experimental results in [5]. The cantilever beam is bonded with a flexible macro fibre composite piezoelectric sensor at the root, as shown in Figure 4 (a). The geometry and material properties of the beam and the MFC sensor can be found in [5]. The clamped beam root is subjected to a sinusoidal base excitation of an acceleration amplitude of $1 \mathrm{~m} / \mathrm{s}^{2}$ at a frequency in the range $23-33 \mathrm{~Hz}$. Firstly, the energy harvesting circuit is connected to a variable resistive load and modelled in the Ansys finite element package, as shown in Figure 4(a). The finite element results of the optimal external resistive load was found to be $400 \mathrm{k} \Omega$ that is equal to analytical and the published result. Then 
the energy harvesting circuit is connected to the optimal resistive load connected between the sensor electrodes and again modelled in the ANSYS finite element package. Figure 4(b) shows the ANSYS finite element results of the harvested energy in the frequency domain. It is the evident that the current results are in a good agreement with the published results of other researchers [5].
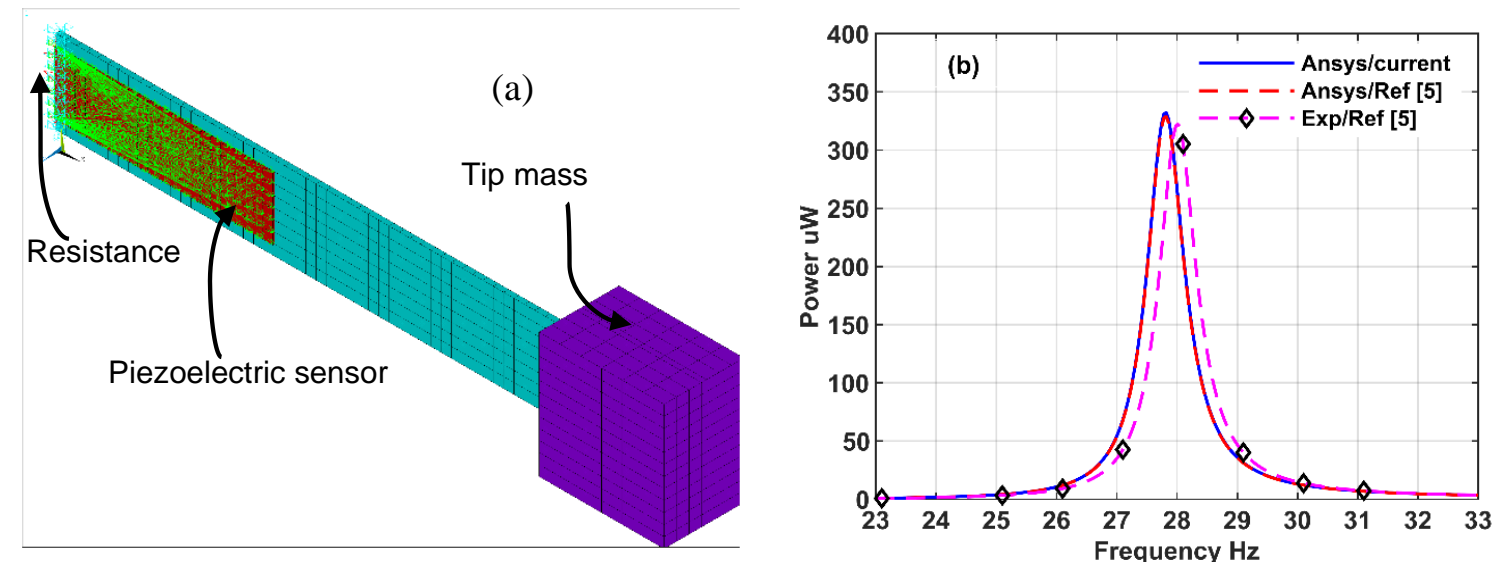

Figure 4.(a) Cantilever beam with tip mass bonded with piezoelectric sensor (current study), (b) Current results of the energy harvesting frequency response at the first mode for a cantilever beam with tip mass under base sinusoidal acceleration excitation of $1 \mathrm{~m} / \mathrm{s}^{2}$ compared to published finite element and experimental tests in [5]

\section{OPTIMISATION OF ENERGY HARVESTING FOR AIRCRAFT WING. 6.1 Optimal sensors placement for aircraft wing.}

Initially, it is assumed that the surface of the composite aircraft wing shown in Fig.2 is fully covered with $22 \times 30$ discrete flexible DuraAct piezoelectric sensors. The dimension of each of the sensor segments is $90 \times 40 \times 0.18 \mathrm{~mm}$, as described in Section 4. The wing was modelled using the Ansys finite element package, as detailed in Sections 2 and 3, to determine the percentage effectiveness of each sensor. The results of the percentage sensor effectiveness distribution over the upper and lower surfaces of the wing are presented in Figure 5. Twenty-five optimal sensor locations on the aircraft wing were chosen based on the ranking stated in Step 6 of Section 3 and are displayed on the upper and lower surface of the wing in Figure 6.

Solving this optimisation problem using a genetic algorithm or similar method is difficult since there are ${ }_{660} \mathrm{C}_{25}\left(>10^{45}\right)$ candidate solutions. On the other hand, using the proposed placement methodology the optimal sensor distribution is obtained quickly with much lower computational effort.
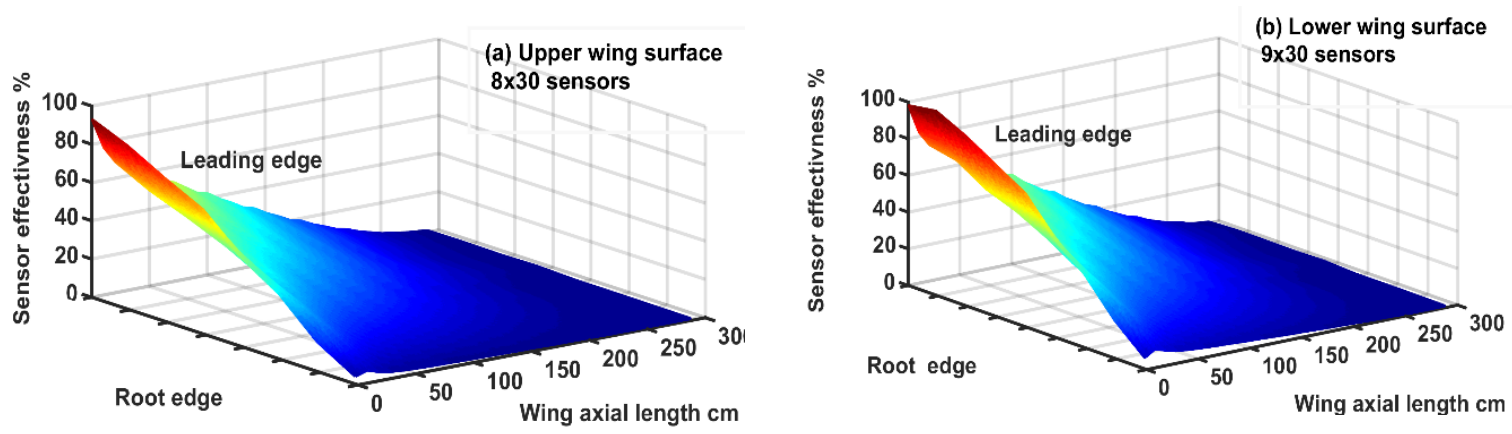

Figure 5. Percentage sensor effectiveness distribution over upper and lower surface of the wing 

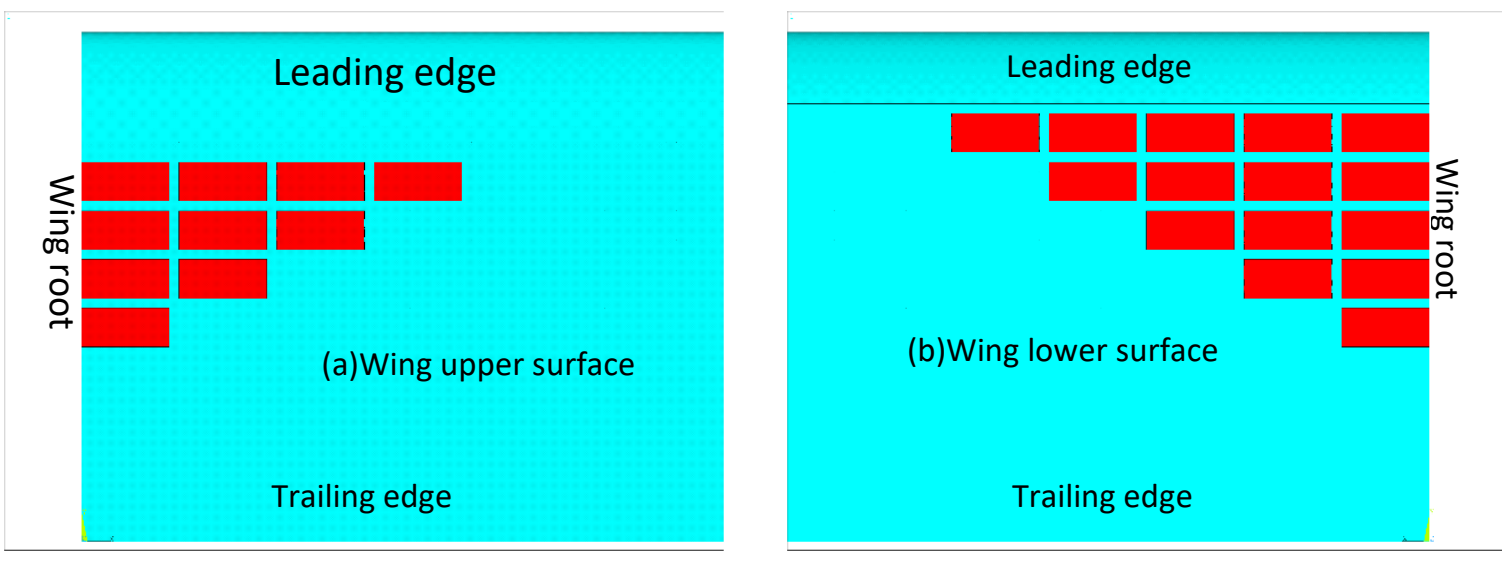

Figure 6. (a) Optimal distribution of ten piezoelectric sensors (S01 to S10) on the upper surface of the wing, and (b) optimal distribution of fifteen piezoelectric sensors (S11-S25) on the lower surface of the wing. The sensors are marked (S01-S25) sequentially increased from the direction of the leading edge to trailing edge and from the root of the wing to the free end.

\subsection{Optimum external resistive load.}

Based on the analytical formula represented in eq. (31), the optimum external resistive load was calculated based on the piezoelectric properties, dimensions and frequency of excitation. The optimum resistance was found to be $75.5 \mathrm{k} \Omega$ at frequency $10 \mathrm{~Hz}$, which is verified by the FE wing model in Ansys. Figure (7) shows Ansys results of the predicted power intensity responses to frequency at different external load resistances. It can be observed from Figure (7) that the maximum harvested power intensity at frequency $10 \mathrm{~Hz}$ occurs at an external load resistance of $75.5 \mathrm{k} \Omega$, which is identical to the prediction from the formula. Furthermore, the power intensity remains consistently high throughout the frequency range $4-10 \mathrm{~Hz}$ if the load resistance is between $75.5 \mathrm{k} \Omega$ and $100 \mathrm{k} \Omega$. The harvesting power value is keeping close to maximum at a certain limit with a range of frequencies and resistances because the voltage increases proportionally with resistance until the voltage reaches a constant value, and the harvesting power is equal to $0.5 \emptyset_{0}^{2} / R$.

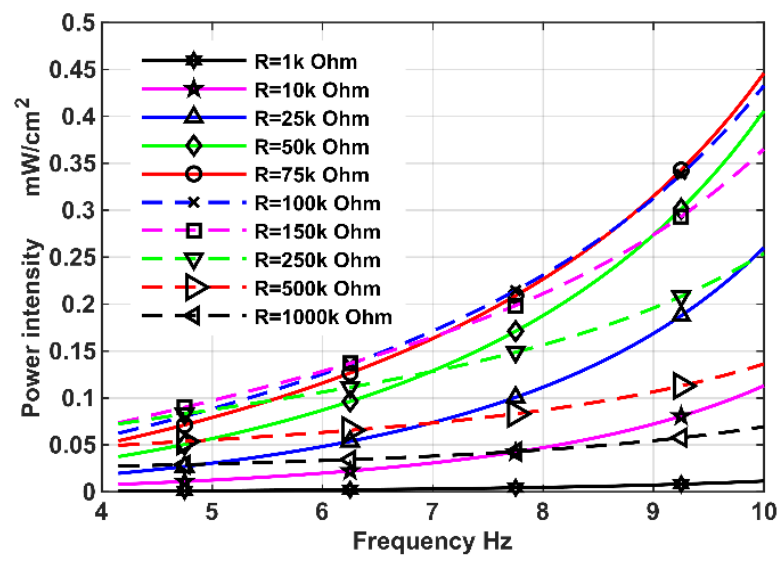

Figure 7. Harvested power intensity frequency response at different values of external load for the piezoelectric sensor (SO1) bonded on the upper surface of the wing. 
Figure 7 shows a large drop in the harvesting power intensity at an external load resistance smaller or larger than the optimal value. Based on eq. (31), the optimal resistance is universally proportional to the value of the piezoelectric capacitance $C_{s}$ and the frequency of vibration $f$. The selection of the proper piezoelectric capacitance at working frequency is crucial to get the optimal resistance matching the real external load resistance and optimise the harvesting power. The value of the piezoelectric capacitor $C_{s}$ is directly proportional to the piezoelectric surface area and piezoelectric permittivity, and universally proportional to the piezoelectric thickness according to the analytical formula represented in eq. (30).

The values of the actual external load resistance and the designed optimal load resistance based on the analytical formula should be identical to optimise the harvesting power. This condition can be achieved by selecting the proper value of piezoelectric sensor capacitance $C_{s}$ based on the dimensions and/or sensor connection in series and parallel.

\subsection{Optimal energy harvesting}

Energy harvesting from the composite aircraft wing connected with the above optimum external load resistance $75.5 \mathrm{k} \Omega$ is studied in this section. The wing is subjected to external disturbances at frequencies in the range 4$10 \mathrm{~Hz}$. Figure (8) shows the electric field distribution over the 25 selected piezoelectric sensor electrodes. The sensors on the upper and lower surfaces illustrate, respectively, negative and positive electric fields, which reflects that the wing is under a bending mode.
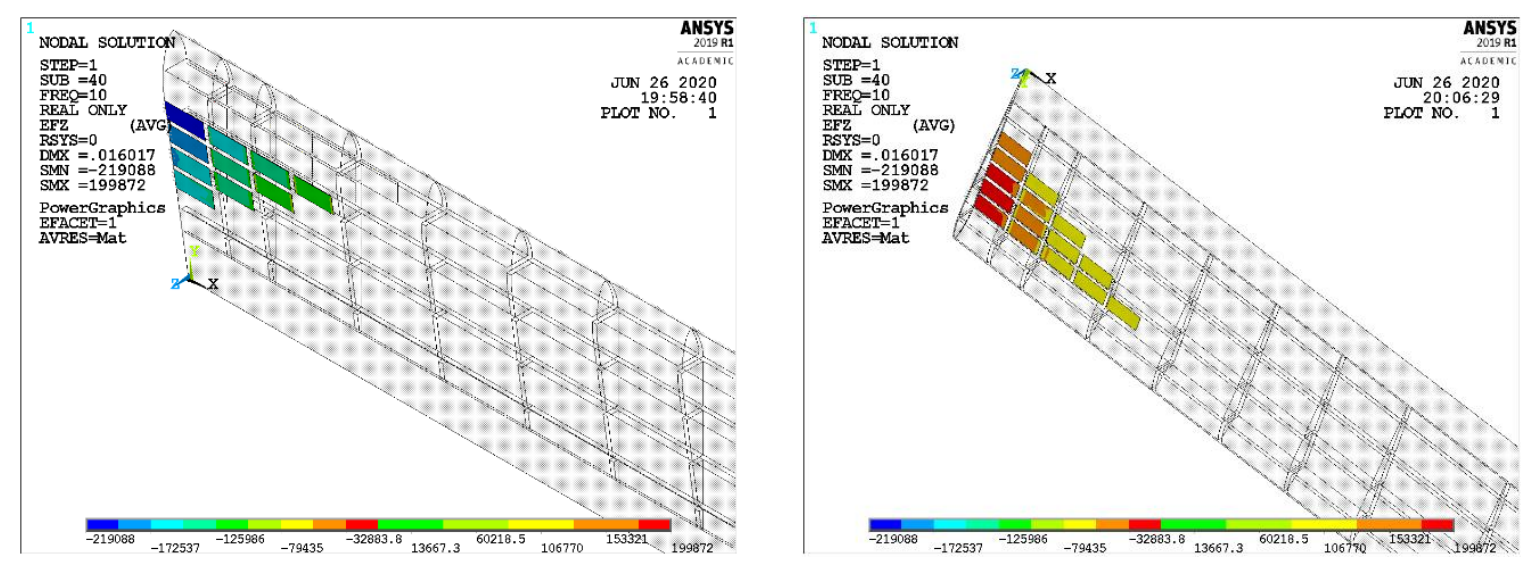

Figure 8. Electric field distribution over piezoelectric surface bonded on the upper and lower wing surfaces.

Figure 9(a) shows strain intensity in the frequency range $4-10 \mathrm{~Hz}$ at the ten optimal sensor locations on the upper wing surface. This shows that the maximum strain intensity at these sensor locations is $210-320 \mu$ at frequency $10 \mathrm{~Hz}$, falling to a range $140-210 \mu$ at $4 \mathrm{~Hz}$. This range of strain and frequencies are within the range of the aircraft structural flight condition $[17,24]$. Figure 9 (b) shows the frequency response of the voltage dropped across the optimal external resistance of $75.5 \mathrm{k} \Omega$. The minimum voltage measured across the resistor is $10 \mathrm{~V}$ at frequency $4 \mathrm{~Hz}$, rising to a maximum of $50 \mathrm{~V}$ at $10 \mathrm{~Hz}$. 

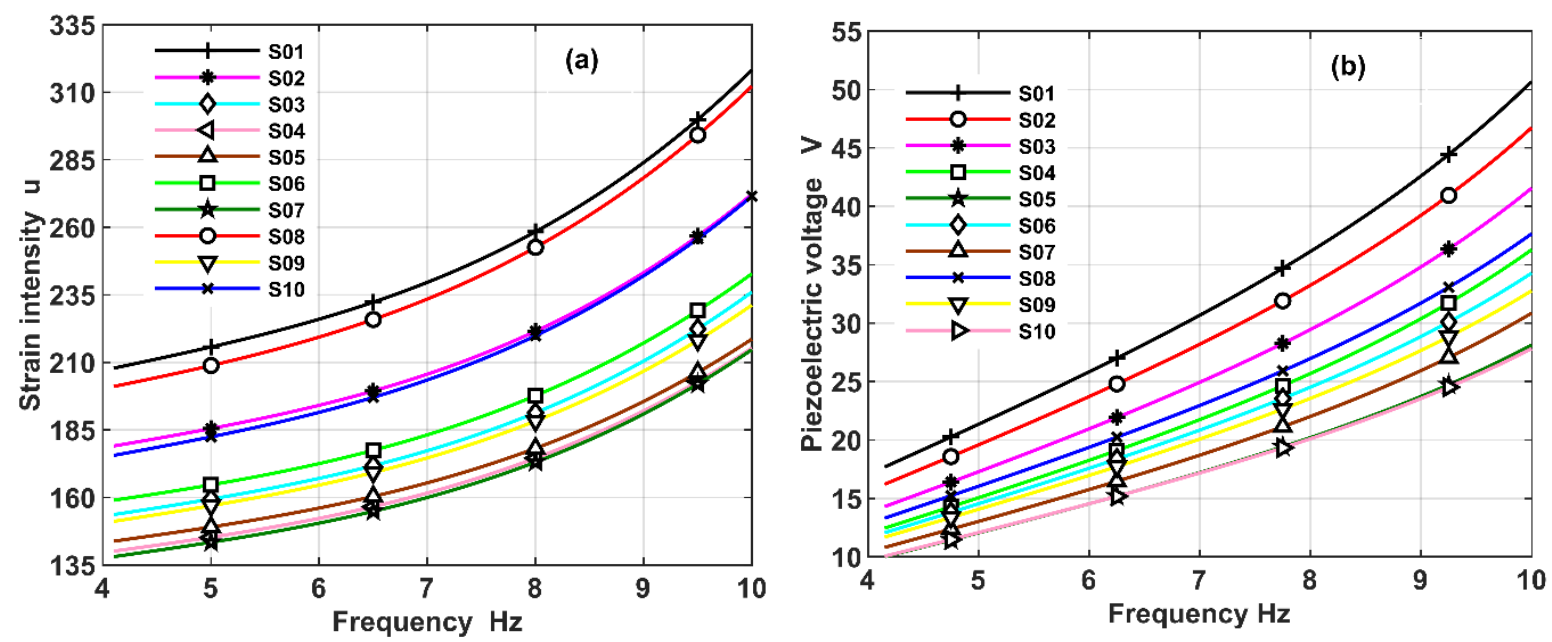

Figure 9. Frequency responses for the ten piezoelectric sensors bonded on the upper surface of the wing. (a) Strain intensities at the locations of the sensors. (b) Voltage dropped over the optimal $75.5 \mathrm{k} \Omega$ load.
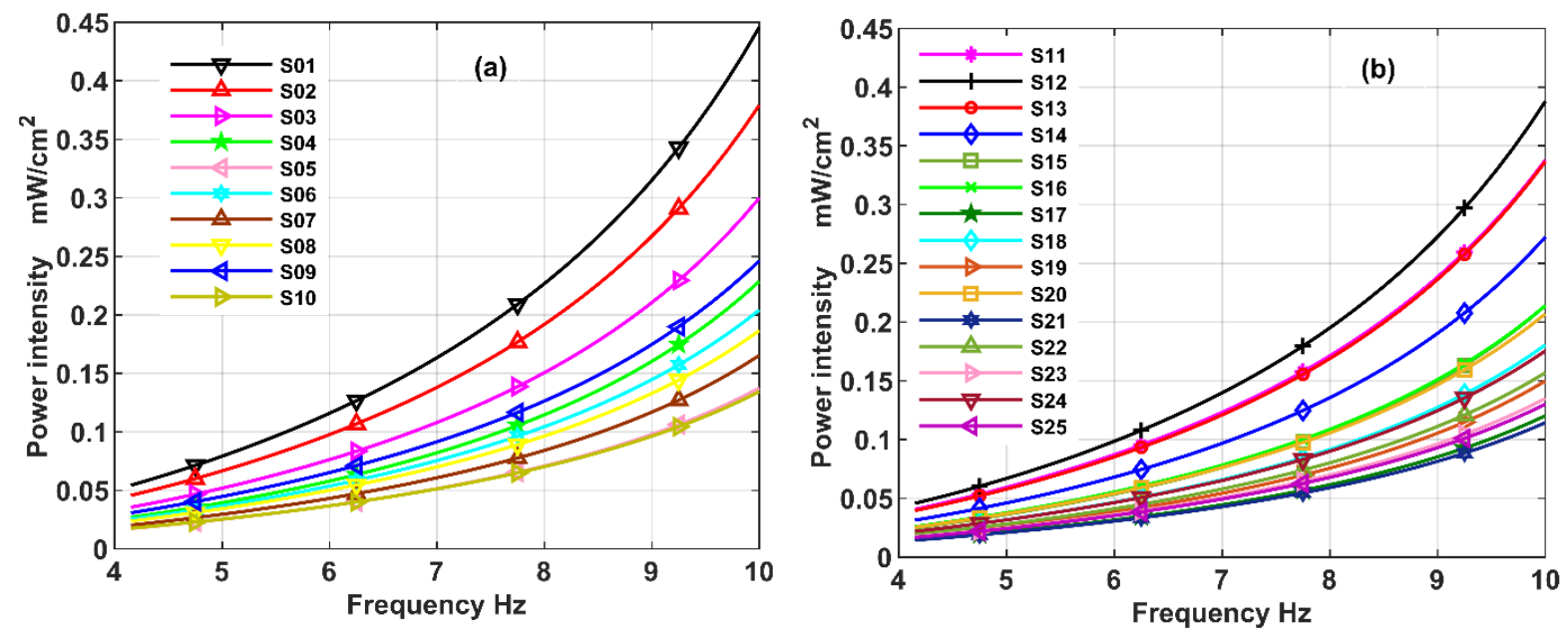

Figure 10. Harvested power intensity frequency responses for the optimally located piezoelectric sensors loaded with the optimal $75.5 \mathrm{k} \Omega$, bonded (a) on the upper surface, and (b) on the lower surface of the wing.

Figures 10(a) and (b) show power intensity frequency responses for the optimally placed sensors bonded to the upper and lower surfaces of the wing, respectively, optimally loaded with $75.5 \mathrm{k} \Omega$. It can be observed from Figure 11 that the minimum harvested power intensity is $0.01 \mathrm{~mW} / \mathrm{cm}^{2}$ at $4 \mathrm{~Hz}$, rising to $0.45 \mathrm{~mW} / \mathrm{cm}^{2}$ at $10 \mathrm{~Hz}$.

The total area of the optimal 25 piezoelectric sensors located on the upper and lower surfaces of the wing is $0.9 \mathrm{~m}^{2}$. From the results in Figure 11, the total sensor area generates electric power of $200 \mathrm{~mW}$ at frequency $10 \mathrm{~Hz}$, falling to $25 \mathrm{~mW}$ at $4 \mathrm{~Hz}$, as shown in Figure 11 . This power is harvested with a peak to peak dynamic strain intensity at the sensor positions in the range $280-640 \mu$. It has been found that the harvested power required for an unmanned aerial vehicle to complete a signal transmission for environmental monitoring is $22.37 \mathrm{~mW}$ 
[26]. The current study indicates that this power can be provided by the 25 optimally located sensors, placed in accordance with the layout obtained by the proposed methodology.

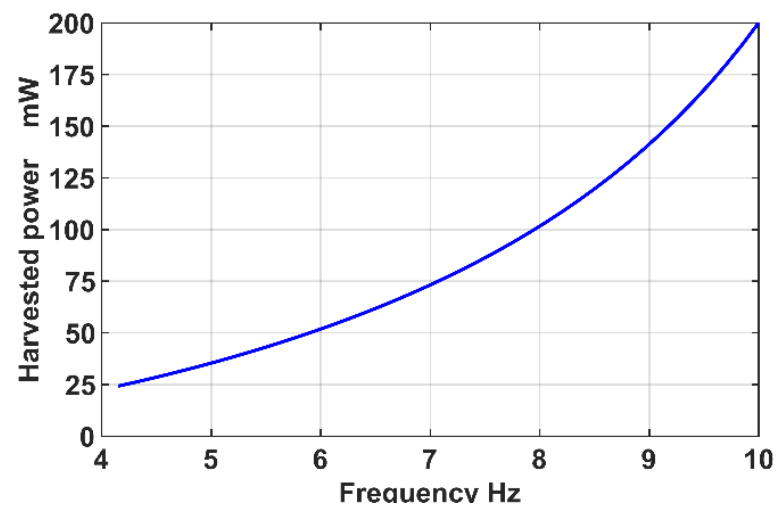

Figure 11. Harvested power frequency responses for all the piezoelectric sensors bonded on the upper surface and lower surface of the wing

\section{CONCLUSION}

This study concerns the optimisation of energy harvesting to power wireless sensor nodes for aircraft structural health monitoring. A generalised placement methodology is proposed to optimise the location of piezoelectric sensors on flexible structures to maximise the voltage and electric power generation. The optimal piezoelectric sensor position is based on the maximisation of the average percentage sensor effectiveness as an objective function. The percentage of sensor effectiveness is a fitness function based on the ratio of sensor voltages normalised to $100 \%$ used to measure the goodness of the sensor position. In this study, energy harvesting is optimised based on the optimal sensor position and electric load resistance, while the dimensions and number of sensors are preselected. The dynamic equilibrium equations were developed for a composite shell stiffened by beams and with bonded piezoelectric sensors connected to an external resistive load, which were then solved using three-dimensional solid finite elements. An optimal sensor placement method was proposed and tested to determine the optimal placement of six piezoelectric sensors for a small composite cantilever plate to maximise the sensor voltage generation at the first five modes of vibration. The results were validated against published work in the literature. It was shown that the proposed method was more efficient than using the genetic algorithms, obtaining an optimal sensor distribution with much reduced computational effort.

The proposed optimal sensor placement method was then applied to optimising the energy harvest sensor locations for an aircraft wing, which would be prohibitively computationally expensive if the genetic algorithm was used, while the current method was shown to be significantly more effective in finding the optimal locations.

The developed energy harvesting model was tested for a cantilever beam with tip mass and bonded with a flexible macro fibre composite sensor. The finite element results of the energy harvesting model was validated against published work. Finally, the placement methodology was employed to find the optimal distribution of flexible DuraAct piezoelectric sensors on an aircraft wing stiffened by four spars and ten ribs. 
It was concluded that the proposed sensor placement method is capable of placing piezoelectric sensors optimally to achieve maximum energy harvest effectively and efficiently due to the simple selection process based on the maximisation of the average percentage sensors effectiveness.

Acknowledgement: The authors at Lancaster University and Coventry University are grateful to the EPSRC for financial support (EP/K020080/1).

\section{REFERENCES}

[1] M. Umeda, K. Nakamura, and S. Ueha, "Analysis of the transformation of mechanical impact energy to electric energy using piezoelectric vibrator," Japanese J. Appl. Physics, Part 1 Regul. Pap. Short Notes Rev. Pap., vol. 35, no. 5 B, pp. 3267-3273, 1996.

[2] H. J. Song, Y.-T. Choi, A. S. Purekar, and N. M. Wereley, "Performance Evaluation of Multi-tier Energy Harvesters Using Macro-fiber Composite Patches," J. Intell. Mater. Syst. Struct., vol. 20, no. 17, pp. 2077-2088, 2009.

[3] R. Patel, S. McWilliam, and A. A. Popov, "A geometric parameter study of piezoelectric coverage on a rectangular cantilever energy harvester," Smart Mater. Struct., vol. 20, no. 8, p. 085004, 2011.

[4] S. C. Stanton, A. Erturk, B. P. Mann, and D. J. Inman, "Nonlinear piezoelectricity in electroelastic energy harvesters: Modeling and experimental identification," J. Appl. Phys., vol. 108, no. 7, pp. 0-9, 2010.

[5] A. Abdelkefi, N. Barsallo, L. Tang, Y. Yang, and M. R. Hajj, "Modeling, validation, and performance of low-frequency piezoelectric energy harvesters," J. Intell. Mater. Syst. Struct., vol. 25, no. 12, pp. 1045389X13507638-, 2013.

[6] Y. Amini, H. Emdad, and M. Farid, "Finite element modeling of functionally graded piezoelectric harvesters," Compos. Struct., vol. 129, pp. 165-176, 2015.

[7] M. Y. Zakaria, M. Y. Al-Haik, and M. R. Hajj, "Experimental analysis of energy harvesting from selfinduced flutter of a composite beam," Appl. Phys. Lett., vol. 107, no. 2, p. 023901, 2015.

[8] Y. Yang, L. Tang, and H. Li, "Vibration energy harvesting using macro-fiber composites," Smart Mater. Struct., vol. 18, no. 11, 2009.

[9] W. G. R. Vieira, C. De Marqui, a Erturk, and D. J. Inman, "Frequency domain piezoaeroelastic analysis and optimisation of an energy harvester wing," Collect. Tech. Pap. - AIAA/ASME/ASCE/AHS/ASC Struct. Struct. Dyn. Mater. Conf., no. April, pp. 1-13, 2010.

[10] C. De Marqui, a. Erturk, and D. J. Inman, "Piezoaeroelastic Modeling and Analysis of a Generator Wing with Continuous and Segmented Electrodes," J. Intell. Mater. Syst. Struct., vol. 21, no. 10, pp. 983-993, 2010.

[11] C. De Marqui, W. G. R. Vieira, A. Erturk, and D. J. Inman, "Modeling and Analysis of Piezoelectric Energy Harvesting From Aeroelastic Vibrations Using the Doublet-Lattice Method," J. Vib. Acoust., vol. 133, no. 1, p. 011003, 2011.

[12] A. H. Daraji and J. M. Hale, "Active vibration reduction by optimally placed sensors and actuators with application to stiffened plates by beams," Smart Mater. Struct., vol. 23, no. 11, p. 115018, 2014.

[13] A. Erturk and D. J. Inman, "On mechanical modeling of cantilevered piezoelectric vibration energy harvesters," J. Intell. Mater. Syst. Struct., vol. 19, no. 11, pp. 1311-1325, 2008.

[14] S. Lee and B. D. Youn, "A new piezoelectric energy harvesting design concept: Multimodal energy harvesting skin," IEEE Trans. Ultrason. Ferroelectr. Freq. Control, vol. 58, no. 3, pp. 629-645, 2011.

[15] V. Balamurugan and S. Narayanan, "Finite element modeling of stiffened piezolaminated plates and shells with piezoelectric layers for active vibration control," Smart Mater. Struct., vol. 19, no. 10, p. 105003, 2010.

[16] F. Frunzulica, A. Dumitrache, M. Stoia, I. Predoiu, and B. Suatean, "Harvesting the wind energy through an actively controlled pitch-plunge flapping wing," Renew. Energy Power Qual. J., vol. 1, no. 14, pp. 1002-1006, 2016.

[17] Y. Shi, S. R. Hallett, and M. Zhu, "Energy harvesting behaviour for aircraft composites structures using macro-fibre composite: Part I - Integration and experiment," Compos. Struct., vol. 160, pp. 1279-1286, 2017.

[18] Y. Cheng, D. Li, J. Xiang, and A. Da Ronch, "Energy harvesting performance of plate wing from discrete gust excitation," Aerospace, vol. 6, no. 3, 2019.

[19] J. Fei, "Active vibration control of a flexible structure using piezoceramic actuators, "Sensors and Tranceducers Journal", Vol. 89, no.3, pp.52-60, 2008. 
[20] H. S. Tzou and C. I. Tseng, "Distributed piezoelectric sensor/actuator design for dynamic measurement/control of distributed parameter systems: A piezoelectric finite element approach," J. Sound Vib., vol. 138, no. 1, pp. 17-34, 1990.

[21] W. Wang, J. Caw, C. R. Bowen, S. Zhou and J. Lin, “Optimum resistance analysis and experimental verification of nonlinear piezoelectric energy harvesting from human motions," Energy. Elsevier Ltd, 118, pp. 221-230, 2017.

[22] S. Ju, S. H. Chae, Y. Choi and C. Ji, "Macro fiber composite-based low frequency vibration energy harvester," Sensors and Actuators, A: Physical. Elsevier B.V., 226, pp. 126-136, 2015.

[23] Covaci, C. and A. Gontean, "Piezoelectric energy harvesting solutions: A review," Sensors (Switzerland), 20(12), pp. 1-37,2020.

[24] Z. J. Chew, T. Ruan, and M. Zhu, "Strain Energy Harvesting Powered Wireless Sensor Node for Aircraft Structural Health Monitoring," Procedia Eng., vol. 168, pp. 1717-1720, 2016.

[25] J.-H. Han and I. Lee, "Optimal placement of piezoelectric sensors and actuators for vibration control of a composite plate using genetic algorithms," Smart Mater. Struct., vol. 8, no. 2, pp. 257-267, 1999.

[26] Marsic V, Giuliano A, Zhu M. Energy autonomous sensing systems based on integrated piezo-fibre composite with carbon-fibre laminates. CDE23681 final report, Cranfield University, 2012. 